\title{
Die größten Steuerfallen für Kardiologen
}

\author{
Major Tax Pitfalls for Cardiologists
}

Autor

Institut
Rolf Michels

Laufenberg Michels und Partner, Köln

\author{
Schlüsselwörter \\ - begünstigte Praxis- \\ veräußerung \\ - Apparategemeinschaften \\ - Abfärbung der gewerblichen \\ Einkünfte \\ - medizinische Heilbehand- \\ lungen in der Umsatzsteuer \\ Key words \\ - tax benefits at sale of a \\ practice \\ - device sharing alliances \\ - municipal trade tax risks \\ - tax-exempt turnovers
}

\section{Was ist wichtig}

- Praxisverkauf: Die steuerlichen Voraussetzungen für eine erhebliche Steuerbegünstigung beim Praxisverkauf lauten wie folgt: Einstellung der beruflichen Tätigkeit nach Verkauf, ein Alter von mehr als 55 Jahren oder dauernde Berufsunfähigkeit und der Verkauf der gesamten Praxis bzw. des gesamten Praxisanteils an einer Gemeinschaftspraxis.

- Steuerliche Risiken bei dem Praxisverkauf: Insbesondere bei der Veräußerung von häuslichen Arbeitszimmern und Praxisimmobilien ist Vorsicht geboten. Steuerlich relevant sind hierbei insbesondere die sog. „stillen Reserven“, die bei einer Veräußerung ohne Zufluss liquider Mittel versteuert werden müssen.

- Separate Veräußerung der Zulassung: Wenn nur eine (Teil-)Zulassung veräußert wird, ist dieser Verkauf grundsätzlich umsatzsteuerpflichtig und bewirkt für alle Beteiligten eine erhebliche Umsatzsteuerbelastung. Eine Ausnahme liegt vor, wenn der Veräußerer ein umsatzsteuerlicher Kleinunternehmer ist. Außerdem unterliegt dieser Verkauf nicht der begünstigen Einkommensteuer, sondern dem normalen Steuersatz.

- Herzkathetermessplatz: Zur Vermeidung steuerlicher Risiken dürfen bei einer Apparategemeinschaft nur Kosten umgelegt werden.

- Kardio-Training: Die Durchführung von Kardio-Trainings kann u. U. umsatzsteuerpflichtig sein.

- Batterieverkauf für Herzschrittmacher: Der Verkauf von Produkten ist gewerbe- und umsatzsteuerpflichtig. Insbesondere bei Gemeinschaftspraxen sollte der Verkauf daher von der Gemeinschaftspraxis getrennt werden.

- Marcumar-Schulungen: Ob Marcumar-Schulungen umsatzsteuerfrei sind, kann nicht mit Gewissheit gesagt werden.

\section{Praxisverkauf}

Ein Kardiologe kann unter bestimmten Umständen Steuerbegünstigungen für den Gewinn aus der Veräußerung seiner Einzelpraxis oder auch seiner Beteiligung an einer Gemeinschaftspraxis in Anspruch nehmen. Bei den Steuerbegünstigungen handelt es sich zum einen um einen steuerlichen Freibetrag und zum anderen um die Begünstigung mit dem „halben“ Steuersatz, bei der der Steuersatz deutlich unter dem Steuersatz für die laufenden Gewinne aus der kardiologischen Tätigkeit liegt. Es gilt hier aber die folgenden Stolperfallen und Hinweise zu beachten.
Voraussetzungen für die Inanspruchnahme der Steuerbegünstigungen

Zunächst sind die Steuerbegünstigungen an bestimmte Voraussetzungen geknüpft [4]. Diese sind

1. Die gesamte Praxis bzw. die gesamte Beteiligung muss unter Aufdeckung sämtlicher stiller Reserven* veräußert werden. Die Veräußerung nur eines Teiles einer Einzelpraxis oder eines Teiles eines Gemeinschaftspraxisanteils ist nicht begünstigt und wie der laufende Gewinn aus der kardiologischen Tätigkeit zu besteuern.

2. Zudem muss der Kardiologe bei Veräußerung das 55. Lebensjahr bereits vollendet haben oder, falls er jünger ist, dauernd berufsunfähig sein. 
3. Die freiberufliche ärztliche Tätigkeit muss unmittelbar mit der Veräußerung für eine gewisse Zeit im örtlichen Wirkungskreis eingestellt werden.

4. Die steuerliche Begünstigung kann nur einmal im Leben beantragt werden.

\section{Hinweise zur ärztlichen Tätigkeit nach Praxisveräußerung}

Die 3. Voraussetzung für die Inanspruchnahme der steuerlichen Begünstigungen ist, dass die freiberufliche ärztliche Tätigkeit unmittelbar mit der Veräußerung für eine gewisse Zeit im örtlichen Wirkungskreis eingestellt wird. Die Begriffe „gewisse Zeit“ und „örtlicher Wirkungskreis“ sind steuerlich nicht einheitlich bestimmt. Die Aufgabe der Tätigkeit für mehr als 3 Jahre wird als ausreichend angesehen, 1 Jahr ist auf jeden Fall zu kurz. Der örtliche Wirkungskreis ist einzelfallbezogen zu prüfen und orientiert sich anhand des Einzugsgebiets der Praxis.

Betroffen von der Einstellung ist jedoch nur die freiberufliche ärztliche Tätigkeit. Eine Fortsetzung der bisherigen Tätigkeit im Angestelltenverhältnis, d. h. als angestellter Arzt, ist unschädlich. Die Voraussetzungen für eine Angestelltentätigkeit auf Lohnsteuerkarte, d.h. der Abschluss eines normalen Anstellungsvertrags mit Weisungsgebundenheit, festem Arbeitslohn, Urlaubsanspruch, Lohnfortzahlung im Krankheitsfall, festen Arbeitszeiten usw., sind jedoch einzuhalten.

Der Bundesfinanzhof hat kürzlich entschieden, dass Verträge über freie Mitarbeit mit dem Erwerber der Praxis, wodurch man freiberuflich für die alte Praxis weiter tätig ist, unschädlich sind. Hier sind aber Risiken (z. B. Scheinselbstständigkeit, Ausweitung der Tätigkeit auf andere Praxen) zu beachten, sodass insofern die Tätigkeit im Anstellungsverhältnis zu empfehlen ist.

Unschädlich wäre es auch, wenn noch in geringem Umfang (d.h. auf jeden Fall weniger als 10\% des bisherigen Umsatzes) Patienten der verkauften Praxis weiter behandelt werden. Jegliche Hinzugewinnung von neuen Patienten ist aber für die steuerliche Begünstigung schädlich. Da hier die Grenzen zu einer Fortsetzung einer „werbenden“ freiberuflichen Tätigkeit häufig schwierig zu ziehen sind, ist hiervon in der Praxis deutlich abzuraten [4].

\section{Hinweise zum Veräußerungsfreibetrag}

Der Veräußerungsfreibetrag beträgt 45000,00€. Er reduziert sich jedoch um den Betrag, um den der Veräußerungsgewinn $136000,00 €$ übersteigt. Das bedeutet, dass ab einen Betrag von $181000,00 €$ kein Freibetrag mehr besteht:

1. Beispiel: Der Veräußerungsgewinn beträgt $140000,00 €$. Der Freibetrag in Höhe von $45000 €$ wird um die Differenz zwischen dem Veräußerungsgewinn, also $140000,00 €$, und dem Betrag in Höhe von 136000,00€ somit um 4000,00€ gemindert. Der Freibetrag beträgt somit in dem Fall $41000,00 €(45000,00 €$ abzüg-

\footnotetext{
* Hinweis: Was sind „stille Reserven“? Von stillen Reserven spricht man, wenn der Verkehrswert höher ist als der steuerliche Buchwert. Beispiel: Ein Arzt hat ein Gerät für $10000,00 €$ angeschafft und davon bereits $8000,00 €$ steuerlich über Abschreibungen geltend gemacht. Dann verbleibt ein restlicher steuerlicher Buchwert für weitere Abschreibungen in Höhe von $2000,00 €$. Das gebrauchte Gerät hat aber noch einen Verkehrswert von 5000,00€, zu dem es verkauft werden könnte. Damit liegt der Verkehrswert von 5000,00€ um 3000,00€ über dem steuerlichen Buchwert von $2000,00 €$. Die 3000,00€ sind stille Reserven, die besteuert werden müssen, wenn das Gerät tatsächlich verkauft würde. Auch der Goodwill einer Praxis, der in der Regel keinen steuerlichen Buchwert hat, stellt stille Reserven einer Praxis dar, die bei Veräußerung besteuert werden müssen. Der Goodwill enthält in der Regel die höchsten stillen Reserven.
}

lich Minderung 4000,00€). Der Veräußerungsgewinn ist in Höhe von $140000,00 €$ abzüglich Freibetrag in Höhe von 41000,00€ nur noch mit 99000,00€ zu versteuern.

2. Beispiel: Der Veräußerungsgewinn beträgt $190000,00 €$. Der Freibetrag wird um 190000,00-136000,00 $=54000,00 €$ auf $0,00 €$ gemindert. Der Veräußerungsgewinn ist in voller Höhe zu versteuern.

\section{Hinweise zum „halben" Steuersatz}

Neben dem Freibetrag gewährt das Steuerrecht, sofern die Voraussetzungen einer begünstigten Veräußerung vorliegen, den sog. halben Steuersatz. Hierzu folgendes Berechnungsbeispiel:

Ein Kardiologe erzielt aus der Veräußerung seiner Praxis einen steuerbegünstigten Veräußerungsgewinn in Höhe von $500000,00 €$. Ein Freibetrag wird aufgrund des hohen Veräußerungsgewinns nicht abgezogen.

Aufgrund der sonstigen laufenden Einkünfte beträgt der durchschnittliche Steuersatz 41,5\% und würde zu einer Einkommensteuer auf den Veräußerungsgewinn in Höhe von 41,5\% auf $500000,00 €=207500,00 €$ führen.

Der sog. halbe Steuersatz reduziert nun den anzuwendenden Steuersatz auf 56\% von 41,5\%, also 23,2\%, und die Einkommensteuer auf den begünstigten Veräußerungsgewinn auf $116000,00 €(23,2 \%$ von $500 \mathrm{~T} €)$, statt $207500,00 €$ ohne Anwendung des halben Steuersatzes.

Die Begünstigung führt hier zu einer Steuerersparnis von $91500,00 €$.

\section{Hinweis zum Veräußerungsstichtag}

Ein weiterer nicht zu vernachlässigender Aspekt bei der Veräußerung der Praxis bzw. des Gemeinschaftspraxisanteils ist die Festlegung des Zeitpunktes der Veräußerung. Ob man seine Praxis am 31.12. oder am darauf folgenden 1.1. veräußert, hat steuerlich unterschiedliche Auswirkungen.

Hintergrund: Die Einkommensteuer ist eine Jahressteuer. Der Einkommensteuer unterliegen die laufenden Einnahmen sowie die erzielten Veräußerungsgewinne, wobei es bei Veräußerungsgewinnen nicht auf den Zeitpunkt der Zahlung des Kaufpreises ankommt, sondern auf den Zeitpunkt der Veräußerung.

Der Einkommensteuertarif ist progressiv. Das bedeutet, je höher die Einkünfte eines Kalenderjahrs sind, desto höher ist der Grenzsteuer- und damit der Durchschnittsteuersatz.

Wählt man als Veräußerungsstichtag den 31.12., fällt der Veräußerungsgewinn mit den laufenden Einkünften aus der Praxis in dasselbe Kalenderjahr und treibt den Steuersatz in die Höhe. In der Regel liegen nach Veräußerung der Praxis nur noch geringere Rentenzahlungen vor, sodass bei einer Veräußerung zum 1.1. der Steuersatz geringer ausfallen kann.

\section{Sonderproblem: Das Arbeitszimmer im eigenen Haus}

In der Vergangenheit wurde vielfach das Arbeitszimmer im eigenen Haus steuerlich geltend gemacht. Hierzu wurden die Anschaffungskosten des eigenen Hauses, sofern sie auf das Arbeitszimmer entfielen, steuerlich abgeschrieben. Das Arbeitszimmer wurde dadurch dem steuerlichen Betriebsvermögen zugeordnet. In der jüngsten Vergangenheit wurde zwar das sog. häusliche Arbeitszimmer aufgrund der hierzu geänderten Rechtslage steuerlich vielfach nicht mehr anerkannt, dies ändert jedoch nichts an der Zuordnung zum Betriebsvermögen.

Das häusliche Arbeitszimmer beinhaltet aufgrund der Abschreibung in der Vergangenheit und der allgemeinen Wertsteigerung 
von Immobilien stille Reserven, die zur Erlangung der Begünstigung besteuert werden müssen.

Hier lauert auch ein weiteres Problem. Zu den Voraussetzungen der begünstigten Besteuerung gehört die Aufdeckung sämtlicher stiller Reserven. Erbringt der Kardiologe nach Beendigung seiner freiberuflichen Tätigkeit als Arzt eine andere freiberufliche Tätigkeit (z. B. als Dozent, Gutachter ...) und nutzt hierfür sein häusliches Arbeitszimmer, bleibt dieses Betriebsvermögen. Die stillen Reserven würden nicht aufgedeckt, die Voraussetzungen der begünstigten Besteuerung wären nicht gegeben.

Der Kardiologe sollte zunächst seine freiberufliche Tätigkeit als Arzt beenden und das häusliche Arbeitszimmer unter Aufdeckung der stillen Reserven in sein Privatvermögen steuerlich überführen. Dadurch sind die Voraussetzungen der begünstigten Besteuerung gegeben.

Mit Aufnahme der anderen freiberuflichen Tätigkeit, wobei sich zur besseren Abgrenzung beide Tätigkeiten nicht überschneiden bzw. aneinander anschließen sollten, wird das häusliche Arbeitszimmer vom Privatvermögen wieder in das neue Betriebsvermögen überführt.

\section{Sonderproblem: Die Praxisimmobilie im Eigentum des Arztes}

Regelmäßig befindet sich die Praxis in von Fremden gemieteten Räumen. Vereinzelt wird die Praxis aber auch in einer Immobilie betrieben, die im Eigentum des Arztes selbst als Einzelpraxisinhaber bzw. als Gesellschafter einer Gemeinschaftspraxis steht. Als Eigentümer nutzt er die Immobilie für seine Einzelpraxis bzw. vermietet sie an die Gemeinschaftspraxis, deren Gesellschafter er ist.

Diese Immobilien wurden vielfach vor langer Zeit gekauft und in der Vergangenheit steuerlich abgeschrieben. Häufig wird in den Fällen zwar die Praxis bzw. der Gemeinschaftspraxisanteil an den Nachfolger veräußert, die Praxisimmobilie verbleibt aber als Geldanlage im Eigentum des abgebenden Arztes. Die Praxisräume werden im Anschluss an den Nachfolger vermietet.

Die Praxisimmobilie beinhaltet aufgrund der Abschreibung in der Vergangenheit und der allgemeinen Wertsteigerung von Immobilien oft hohe stille Reserven, die zur Erlangung der Begünstigung besteuert werden müssen.

Nachteil dieser Besteuerung ist, dass der Praxisverkäufer die stillen Reserven zum Zeitpunkt der Praxisveräußerung nicht realisiert, also keinen Kaufpreis für die Immobilie erhält, sie jedoch trotzdem versteuern muss. Dies kann zu Liquiditätsproblemen führen.

Der Vorteil ist jedoch, dass nach Überführung der Praxisimmobilie ins Privatvermögen zukünftige Wertsteigerungen (nach Ablauf einer 10-jährigen Spekulationsfrist für Immobilienverkäufe) bei einer Veräußerung nicht mehr versteuert werden müssen.

Die Besteuerung der stillen Reserven in der Praxisimmobilie kann vermieden bzw. langfristig in die Zukunft vertagt werden, wenn die Praxisimmobilie in eine neu gegründete $\mathrm{GmbH} \& \mathrm{Co}$. KG ohne Aufdeckung der stillen Reserven übertragen wird.

Der Vorteil ist, dass zum Zeitpunkt der Praxisaufgabe die stillen Reserven nicht versteuert werden müssen.

Nachteilig hingegen ist, dass zukünftige Wertsteigerungen steuerbehaftet sind, sodass eine spätere Veräußerung auch nach Ablauf von 10 Jahren steuerpflichtig ist. Die Verwaltungskosten einer $\mathrm{GmbH}$ \& Co. KG sind ebenfalls nicht zu unterschätzen. Es sind jährlich Jahresabschlüsse für die KG und die $\mathrm{GmbH}$ zu erstellen und offenzulegen.
Des Weiteren besteht stets das Risiko, dass die Finanzverwaltung diese Gestaltung als Gesamtplan zur Verringerung der Steuerbelastung ansieht und das Konstrukt steuerlich nicht anerkennt. Hier bedarf es frühzeitiger Planungen, die zeitlich einige Jahre vor der eigentlichen Praxisveräußerung liegen sollten.

\section{Abschreibung des Praxiserwerbers}

Der Erwerber der Praxis kann den Kaufpreis, den er für die Praxis zahlt, über Abschreibungen zeitlich versetzt steuerlich geltend machen [3]. Der Kaufpreis wird hierbei auf die verschiedenen erworbenen Wirtschaftsgüter (Goodwill, Geräte, Einrichtungen etc.) verteilt, wobei der erworbene Goodwill, d.h. der immaterielle Wert, meist von größter Bedeutung sind.

Auch dieser Goodwill kann gewinnmindernd in den kommenden Jahren abgeschrieben werden. Die Rechtsprechung nimmt an, dass sich der Goodwill einer Einzelpraxis im Laufe von 3-5 Jahren und ein Goodwill einer Gemeinschaftspraxis im Laufe von 6-10 Jahren verflüchtigen werden. Kauft sich ein Kardiologe z.B. in eine Gemeinschaftspraxis ein und zahlt für den Goodwill $400000,00 €$, kann er pro Jahr ein Achtel, also pro Jahr $50000,00 €$ zur Reduzierung seiner Steuerbelastung als Abschreibung geltend machen.

Im Regelfall sind kürzere Abschreibungsdauern stets von Vorteil, da sie die Steuerbelastung mindern. Im Rahmen von Praxiskäufen ist jedoch zu empfehlen, anhand von Planungsrechnungen zunächst zu kalkulieren, wie sich der Gewinn in den ersten Jahren entwickeln wird. Es kann in diesen Fällen steuerlich sinnvoll sein, längere Abschreibungszeiträume zu wählen, da erst später mit hohen Gewinnen zu rechnen ist.

\section{Kurz gefasst.}

Ist ein Kardiologe älter als 55 Jahre, veräußert seine gesamte Praxis bzw. seinen gesamten Anteil an einer Gemeinschaftspraxis und stellt seine freiberufliche ärztliche Tätigkeit ein, kann er einmal im Leben für den dabei entstehenden Veräußerungsgewinn die Steuervergünstigung des „halben“ Steuersatzes und ggf. des Veräußerungsfreibetrags in Anspruch nehmen. Dies führt zu einer deutlichen Reduzierung der Steuerbelastung.

\section{Verkauf nur der (Teil-)Zulassung}

$\nabla$

Verkauft ein Kardiologe nur seine (Teil-)Zulassung, so wird der Gewinn hieraus nicht begünstigt bei der Einkommensteuer besteuert, weil er nicht seine gesamte Praxis veräußert.

Neben der Belastung mit Einkommensteuer zum „normalen“ Steuersatz kommt eine mögliche Belastung mit Umsatzsteuer hinzu.

Grundsätzlich unterliegt jede Lieferung oder Dienstleistung eines Unternehmers der Umsatzsteuer. Das Gleiche gilt auch für Ärzte. Die Dienstleistungen der Ärzte werden jedoch, soweit es sich um Heilbehandlungen mit einem therapeutischen Ziel handelt, von der Umsatzsteuer befreit.

Das Gleiche gilt z. B. für den Verkauf des Praxisinventars. Umsatzsteuerlich handelt es sich hierbei um die Lieferung eines Gegenstands. Hierfür gilt eine gesonderte Steuerbefreiungsvorschrift für derartige Lieferungen. Voraussetzung ist, dass der Arzt den Gegenstand für seine Heilbehandlungen verwendet hat.

Veräußert der Kardiologe nun seine (Teil-)Zulassung, fällt dies nicht unter die Steuerbefreiung für Heilbehandlungen, weil es 
sich bei der Veräußerung nicht um die medizinische Betreuung von Menschen mit therapeutischem Ziel handelt.

Es fällt jedoch auch nicht unter die Steuerbefreiung für Lieferungen, da der europäische Gerichtshof entschieden hat, dass die Veräußerung der Zulassung als Teil des Goodwills eine Dienstleistung darstellt. Damit fällt grundsätzlich Umsatzsteuer auf den Verkaufspreis der Zulassung an.

Einen Ausweg kann die sog. Kleinunternehmerregelung bieten. Umsatzsteuer entsteht grundsätzlich nur, wenn die gesamten steuerpflichtigen Umsätze im Vorjahr 17500,00 $€$ und im laufenden Jahr 50000,00€ überschreiten. Sofern die steuerpflichtigen Umsätze diese Grenzen nicht erreichen, greift die sog. Kleinunternehmerregelung, sodass keine Umsatzsteuer entsteht. Da Heilbehandlungen und der Verkauf von Gegenständen, die für Heilbehandlungen verwendet wurden, nicht steuerpflichtig sind, kann, sofern der Kardiologe keine umsatzsteuerpflichtigen Umsätze tätigt, die Kleinunternehmerregelung greifen.

\section{Kurz gefasst}

Der Verkauf der (Teil-)Zulassung kann zu einer ungewollten Umsatzsteuerbelastung führen, sofern die Kleinunternehmerregelung nicht greift.

\section{Herzkathetermessplatz \\ $\nabla$}

Aufgrund des technischen Fortschritts wurden für die Medizin in den vergangenen Jahren eine Vielzahl medizinischer Großgeräte und Hilfsmittel entwickelt. Patienten fordern, auf dem aktuellsten Stand der medizinischen und technischen Entwicklung betreut zu werden. Für Ärzte bedeutet dies neben einer ständigen persönlichen Fortbildung u. a. auch kostspielige Investitionen für diverse Großgerätschaften. Um eine solche Behandlung wirtschaftlich lukrativ anbieten zu können, schließen sich häufig vermehrt Ärzte zu sog. Apparategemeinschaften - z. B. zur Nutzung eines Herzkathetermessplatzes - zusammen, um sich die Kosten der Anschaffung teilen zu können.

Diese sog. Apparategemeinschaften werden meist in der Rechtsform der Gesellschaft bürgerlichen Rechts (GbR) gegründet. Sie sind nicht auf Gewinnerzielungsabsicht ausgerichtet, sondern legen die entstandenen Kosten nach einem bestimmten Schlüssel auf die einzelnen Gesellschafter um [1].

Der Behandlungsvertrag kommt nicht zwischen der Apparategemeinschaft, sondern zwischen dem behandelnden Arzt und den Patienten zustande. Das Honorar steht somit weiterhin dem Arzt zu.

Steuerlich gilt es dabei zur Vermeidung von Gewerbe- und Umsatzsteuer folgende Punkte zu beachten:

Auf Ebene der Apparategemeinschaft darf kein Gewinn erzielt werden, da ansonsten die Apparategemeinschaft gewerblich tätig ist und Einkünfte aus Gewerbebetrieb erzielt, die der Gewerbesteuer unterliegen [2]. Das bedeutet, dass die Apparategemeinschaft ihren Gesellschaftern nur die anteiligen Kosten weiterberechnen darf. Bei einem Herzkathetermessplatz sollte sich die Verteilung z. B. anhand der erfolgten Nutzungen orientieren.

Unterjährig können die Nutzungen in der Form weiterbelastet werden, dass bei Nutzung die voraussichtlichen Kosten vom Nutzer an den Herzkathetermessplatz erstattet werden. Steuerlich handelt es sich hierbei nicht um Einnahmen der Apparategemeinschaft, sondern um sog. Einlagen ohne Gewinnauswir- kung. Am Ende des Jahres werden die anteiligen Kosten der einzelnen Kardiologen als Verluste den Beteiligten anhand der jeweiligen Inanspruchnahme zugewiesen.

Wird der Herzkathetermessplatz an Dritte überlassen, kann dies zur Gewerblichkeit und somit zur Gewerbesteuer führen.

Liegt bspw. eine Apparategemeinschaft zwischen niedergelassenen Ärzten (in einer Gemeinschaftspraxis) und einem Krankenhaus vor, kann die Überlassung an Dritte über das Krankenhaus erfolgen. Das Krankenhaus stellt einem Dritten den Herzkathetermessplatz zur Verfügung und muss an die Apparategemeinschaft die anteiligen Kosten aufgrund der Nutzung als eigene Nutzung erstatten. Die Apparategemeinschaft selbst darf den Herzkathetermessplatz nicht an einen Dritten überlassen.

Ist als weiterer Beteiligter neben dem Krankenhaus eine kardiologische Gemeinschaftspraxis beteiligt, so empfiehlt sich zur Vermeidung einer gewerblichen Prägung der Gemeinschaftspraxis, dass sich die Ärzte direkt an der Apparategemeinschaft beteiligen und nicht die kardiologische Gemeinschaftspraxis als solche. Kommt es ungewollt zu gewerblichen Einkünften der Apparategemeinschaft, führt dies nicht zu einer Infizierung der kardiologischen Gemeinschaftspraxis.

Umsatzsteuerlich gilt Folgendes: Sofern lediglich die Kosten des Herzkathetermessplatzes nach dem Verhältnis der Beanspruchung auf die Gesellschafter aufgeteilt werden, werden diese Leistungen des Herzkathetermessplatzes von der Umsatzsteuer befreit, da sie unmittelbar von den beteiligten Ärzten zur Ausführung ihrer steuerfreien Heilbehandlungen verwendet werden.

\section{Kurz gefasst}

Zur Vermeidung einer Gewerbe- und Umsatzsteuerbelastung dürfen nur die anteiligen Kosten an die Mitglieder weiterbelastet werden. Apparategemeinschaften sollten ihre Leistungen nur an ihre Mitglieder erbringen. Sofern Leistungen an Dritte erbracht werden, sind steuerliche Gestaltungen gefragt.

\section{Kardio-Training}

$\nabla$

Es kann je nach Krankheitsbild medizinisch sinnvoll sein, das Herz-Kreislauf-System gezielt zu belasten. Einige Kardiologen bieten nunmehr im Anschluss an durchgeführte Heilbehandlungen ihren Patienten ein ambulantes Training an. Dieses Training umfasst in der Regel ein abgestimmtes Ausdauertraining. Im Vergleich zu herkömmlicher Krankengymnastik geht es nicht um Muskelaufbau, sondern um Konditionstraining. Die Trainingseinheiten werden nicht in Form von Einzeltrainerstunden gegeben, sondern erfolgen im Rahmen von Gruppenveranstaltungen.

Sofern dieses Konditionstraining im Rahmen einer laufenden Heilbehandlung erfolgt, wird es als Teil der ärztlichen Heilbehandlung von der Umsatzsteuer befreit.

Vielfach besuchen jedoch auch Teilnehmer dieses Angebot, deren ärztliche Heilbehandlung abgeschlossen ist bzw. die nicht Patienten der Praxis sind. Bei diesen Teilnehmern erfolgt die Leistung nicht als Teil einer Heilbehandlung. Erfolgt die Leistung gegenüber solchen Teilnehmern, greift die Umsatzsteuerbefreiungsvorschrift für ärztliche Heilbehandlungen nicht, sodass die Leistung mit Umsatzsteuer abzurechnen ist. Die Umsatzsteuer ist an das Finanzamt abzuführen. Ob der Teilnehmer ein sog. „Selbstzahler" ist oder z. B. die Krankenkasse die Kosten im Rahmen der 
Primärprävention gem. §20 SGB V trägt, spielt für die Beurteilung keine Rolle.

Sofern die Kleinunternehmerregelung (a.a.o.) nicht greift und die steuerpflichtigen Umsätze über $17500,00 €$ liegen, sollte die Umsatzsteuer in die Preiskalkulation einbezogen werden, damit sie nicht zu einem echten Kostenfaktor wird.

Sofern die Leistungen außerhalb von medizinischen Heilbehandlungen ohne therapeutischen Zweck erbracht werden, handelt es sich ebenso wie beim medizinischen Gerätetraining ohne ärztliche Verordnung um gewerbliche Einkünfte. Der Kardiologe tritt hierbei in Wettbewerb mit gewerblichen Fitnessstudios. Sofern diese gewerblichen Umsätze in nicht nur geringen Umfang erfolgen, droht zudem die gewerbliche Infizierung der übrigen Einnahmen, sofern das Kardio-Training von Ärzten erbracht wird, die sich zu einer Personengesellschaft zusammengeschlossen haben.

\section{Kurz gefasst.}

Beim Kardio-Training gilt es zu unterscheiden, ob die Leistung an Patienten im Rahmen eines Therapieplans erfolgt oder von Dritten besucht wird.

\section{Batterieverkauf für Herzschrittmacher (Produktverkauf) \\ $\nabla$}

Grundsätzlich erzielen Kardiologen, ob in einer Einzelpraxis oder in einer Gemeinschaftspraxis tätig, Einkünfte aus freiberuflicher Tätigkeit, die nur der Einkommensteuer, aber nicht der Gewerbesteuer unterliegen.

Verkaufen z.B. Kardiologen in ihren Praxen Batterien für Herzschrittmacher, so handelt es sich hierbei jedoch um eine gewerbliche Tätigkeit. Gewerbliche Einkünfte unterliegen der Gewerbesteuer.

Kardiologen in Einzelpraxen können neben Einkünften aus freiberuflicher Tätigkeit Einkünfte aus dem Gewerbebetrieb erzielen. Die Einkünfte können getrennt ermittelt werden, sodass lediglich die Einkünfte aus dem Verkauf der Batterien der Gewerbesteuer unterliegen. Die Gewerbesteuer gewährt einen jährlichen Freibetrag von 24500,00€, sodass regelmäßig nicht mit einer Gewerbesteuerbelastung zu rechnen ist.

Verkauft hingegen eine Gemeinschaftspraxis an ihre Patienten Batterien für Herzschrittmacher, so besteht bei Gemeinschaftspraxen die Gefahr, dass sämtliche Einkünfte der Praxis in gewerbliche Einkünfte umqualifiziert werden und der gesamte Praxisgewinn der Gewerbesteuer unterliegt. Aufgrund der teilweisen gewerblichen Einnahmen kann es zu einer Abfärbung der Gewerblichkeit auf die regulären Praxiseinnahmen kommen. Sind die gewerblichen Einkünfte jedoch nur geringfügig, entfällt eine Abfärbung. Eine gewerbliche Abfärbung ist laut Rechtsprechung zu verneinen, wenn der Verkauf von Batterien, also der gewerbliche Umsatz, weniger als 1,25\% des Gesamtumsatzes ausmacht. Erst wenn mit den gewerblichen Einnahmen mehr als 1,25\% des Gesamtumsatzes erwirtschaftet werden, führt dies zu einer Abfärbung auf die übrigen Einkünfte, womit sämtliche Einkünfte aus der Gemeinschaftspraxis in gewerbliche Einkünfte umqualifiziert werden. Der gewerbesteuerliche Freibetrag von $24500,00 €$ ist an dieser Stelle nicht mehr hilfreich, da die Einkünfte einer Gemeinschaftspraxis regelmäßig wohl 24500,00€ übersteigen.
Die Gewerbesteuer wird allerdings auf die Einkommensteuer angerechnet. Das mildert den Gewerbesteuereffekt, sodass die endgültige Belastung mit Gewerbesteuer, bei Ärzten im einkommensteuerlichen Spitzensteuersatz, bei ca. 2\% der Einkünfte liegt, im Extremfall aber auch bis zu 15\% des Gewinns betragen kann.

Die gewerbliche Infizierung kann bei Gemeinschaftspraxen vermieden werden, indem im Vorfeld eine weitere gesonderte beteiligungsidentische Gemeinschaftspraxis gegründet wird, welche die gewerblichen Einkünfte unabhängig von der kardiologischen Gemeinschaftspraxis tätigt, sodass der Verkauf nicht die Gemeinschaftspraxis infizieren kann.

Der Verkauf von Batterien unterliegt in der Regel auch der Umsatzsteuer, sofern die Kleinunternehmerreglung nicht zum Tragen kommt.

Kurz gefasst

Ohne vorherige Gestaltung kann der Verkauf von Batterien oder anderer Produkte zu einer ungewollten Gewerbesteuerbelastung der gesamten Praxiseinkünfte führen, wenn diese Tätigkeit in der Gemeinschaftspraxis nicht im Vorfeld von dem gewerblichen Verkauf der Batterien getrennt wird.

\section{Marcumar-Schulungen}

$\nabla$

Patienten, die Marcumar regelmäßig einnehmen, müssen zur Kontrolle der Dosierung in regelmäßigen Abständen ihre Blutwerte untersuchen lassen. Die Krankenkassen geben diesen Patienten die Möglichkeit, die Höhe der Dosierung selbst zu bestimmen. Hierzu erhalten die Patienten, vergleichbar mit einem Blutzuckermessgerät, ein entsprechendes Gerät, das von den Krankenkassen finanziert wird.

Voraussetzung, um ein solches Gerät zu erhalten, ist, dass der Patient nachweisen kann, dass er an einer entsprechenden Schulung im Umgang mit dem Gerät teilgenommen hat. Die Kosten für diese Schulung werden teilweise von den Krankenkassen übernommen.

Einige Kardiologen erteilen nunmehr ihren Patienten als besonderen Service entsprechende Schulungen, damit diese das Gerät erhalten können.

Umsatzsteuerlich stellt sich das Problem, dass nicht eindeutig feststeht, ob es sich um eine umsatzsteuerfreie Leistung handelt. Damit eine medizinische Leistung umsatzsteuerfrei ist, muss sie grundsätzlich folgende Voraussetzungen [6] erfüllen:

- Es muss sich um eine Leistung im Bereich der Humanmedizin handeln,

- die Leistung muss in der Regel durch einen Arzt erfolgen und

- die Leistung verfolgt ein therapeutisches Ziel. Hierzu zählen Leistungen, die der Diagnose oder der Behandlung von Krankheiten und anderen Gesundheitsstörungen, sowie Leistungen, die dem Schutz und der Aufrechterhaltung der Gesundheit dienen.

Es steht zweifellos fest, dass die korrekte Bedienung eines solchen Geräts eine Grundvoraussetzung für den Patienten ist, damit dieser anschließend die Marcumar-Dosierung in Eigenregie richtig festlegen kann.

Man kann den Standpunkt vertreten, dass die Schulung dem Schutz und der Aufrechterhaltung der Gesundheit dient.

Neben dem medizinischen Nutzen führt die Nutzung des Geräts zu einer Verbesserung der Lebensqualität des Patienten, da dieser nicht mehr in kurzen regelmäßigen Abständen seinen Arzt auf- 
suchen muss, sondern eigenständig die Dosierung festlegt. Ein solches Gerät ist medizinisch nicht zwingend erforderlich.

Die Tatsache, dass die Kosten für diese Schulung teilweise von den gesetzlichen Krankenkassen übernommen werden, ist ein Indiz dafür, dass es sich um eine steuerfreie Leistung handelt. Wie diese Leistungen umsatzsteuerlich zu würdigen sind, ist bisher nicht durch die Finanzverwaltung bzw. Gerichte geklärt worden. Sofern die Kleinunternehmerregelung nicht zum Tragen kommt, könnte Umsatzsteuer entstehen.

Kurz gefasst

Es kann an dieser Stelle nicht mit Gewissheit gesagt werden, dass die Marcumar-Schulungen umsatzsteuerfrei sind.

\section{Fazit}

Steuerlich gelten stets Besonderheiten, die zu beachten sind, sofern die Steuerbelastung möglichst gering ausfallen soll. Die steuerliche Gestaltung sollte noch vor Beginn des Handelns ansetzen, damit man sich nicht in der rettenden Beratung wieder findet.

\section{Zusammenfassung}

$\nabla$

Wenn die Voraussetzungen zur steuerlich begünstigten Veräußerung der Praxis vorliegen, kann die Einkommensteuerbelastung gesenkt werden. Die Einkommensteuerbelastung lässt sich durch nicht spürbare Maßnahmen, wie z. B. das Verlegen des Veräußerungsstichtags vom 31.12. auf 1.1., noch weiter senken.

Durch eine nicht durchdachte Planung kann bei einer Apparategemeinschaft, z.B. wie beim Herzkathetermessplatz, ungewollt und unnötig eine Umsatzsteuerbelastung entstehen.

Die Gewerbesteuer kann auf sämtliche Einkünfte entfallen, sofern keine Trennung der Einkünfte durch eine entsprechende Gestaltung wie z.B. beim Verkauf von Batterien und der Leistungserbringung einer Apparategemeinschaft gelingt.

\section{Abstract}

If the requirements for tax advantages relating to the sale of a practice are given, this can reduce the income tax burden. This burden can be further reduced by measures of minor significance such as fixing the sale date on 01.01. rather than 31.12.

If a plan is not well considered, this can cause an unwanted and unnecessary turnover tax burden for a device sharing alliance such as a heart catheter measuring station.

Trade tax may apply to all income if this income cannot be separately taxed due to optimised fiscal planning such as in the case of the sale of batteries and the provision of services by a device sharing alliance.

\section{Interessenkonflikt}

Der Autor gibt an, dass er in keinem Interessenkonflikt steht.

\section{Literatur}

1 Peikert P. In: Rieger H-J, Dahm F-J, Steinhilper G, Hrsg. Heidelberger Kommentar Arztrecht Krankenhausrecht Medizinrecht, 150 - Apparategemeinschaft. Heidelberg: C. F. Müller; 2002

2 Michels R, Möller K-H. Ärztliche Kooperationen. 2. Aufl. Herne: nwb; 2010

3 Michels R. Abschreibungen auf den Praxiswert unter Berücksichtigung der aktuellen Rechtsprechung. GesR 2012

4 Michels R, Ketteler-Eising T. Risiken und Gestaltungsmöglichkeiten bei der Einstellung der ärztlichen Tätigkeit. Praxis Freiberufler-Beratung 2007

5 Michels R. Steuerliche Aspekte der Praxisabgabe. ZWD 2011

6 Klaßmann R, Lewejohann D, Offermanns H et al. Besteuerung der Ärzte, Zahnärzte und sonstiger Heilberufe. 8. Aufl. Herne: nwb; 2013 